\title{
Effects of contrast medium exposure on urine albumin/ creatinine ratio
}

\author{
@Danem Kayhan, @Fatih Kıvrakoğlu, @Ayşenur Miray Yarlığlu, @Mercan Tastemur, \\ (1)Seyit İbrahim Akdağ \\ University of Health Sciences, Dışkapı Yıldırım Beyazıt Training and Research Hospital, Department of Internal Medicine, Ankara, Turkey
}

Cite this article as: Kayhan S, Kıvrakoğlu F, Yarlığlu AM, Tastemur M, Akdağ Sİ. Effects of contrast medium exposure on urine albumin/ creatinine ratio. J Health Sci Med 2022; 5(1): 49-53.

\begin{abstract}
Aim: Albuminuria is a direct consequence of renal glomerular injury and increases with glomerular dysfunction. Spot urine albumin/creatinine $(\mathrm{Alb} / \mathrm{Cr}$ ) ratio is a reasonable surrogate for 24-hour urine albumin excretion rate and certainly not without limitations. It is known that renal function can be affected following contrast agent administration. The aim of our study is to assess the changes in $\mathrm{Alb} / \mathrm{Cr}$ ratio in spot urine before and after contrast agents in patients undergoing computed tomography (CT) scanning.

Material and Method: The present study included 103 hospitalized patients aged between 18 and 75 years, who underwent contrast-enhanced CT scanning for any reason and did not develop contrast-induced nephropathy (CIN). We compared the values of $\mathrm{Alb} / \mathrm{Cr}$ ratio at the $6^{\text {th }}, 12^{\text {th }}, 24^{\text {th }}, 48^{\text {th }}$, and $72^{\text {nd }}$ hours after the procedure (post-procedure time) with the values at pre-procedure time.

Results: The median age of the patients were 61 years. It has been observed that there is no significant increased in microalbuminuria after the use of contrast media. When the patients were evaluated for the albuminuria level before the procedure, it has been seen that 73 patients (70.9\%) had an Alb/Cr ratio of $<30 \mathrm{mg} / \mathrm{g}$ (group-1) and 30 patients (29.1\%) had an $\mathrm{Alb} / \mathrm{Cr}$ ratio of $\geq 30 \mathrm{mg} / \mathrm{g}$ (group-2). In group 1, it has been observed that the $\mathrm{Alb} / \mathrm{Cr}$ ratios at the post-procedure $6^{\text {th }}, 12^{\text {th }}$, $24^{\text {th }}$, and $48^{\text {th }}$ hours were statistically significantly higher than the value at pre-procedure time. In group 2 , it has been observed that $\mathrm{Alb} / \mathrm{Cr}$ ratio values at all post-procedure time except the $24^{\text {th }}$ hour were statistically significantly lower than the values at the pre-procedure time.

Conclusion: It should be considered that there might be changes in $\mathrm{Alb} / \mathrm{Cr}$ ratio even without developing significant complications such as CIN in patients exposed to contrast medium.
\end{abstract}

Keywords: Contrast medium, urine albumin/creatinine ratio, microalbuminuria

\section{INTRODUCTION}

Contrast-induced nephropathy (CIN) is an important complication that develops as a result of exposure to contrast media during diagnostic procedures such as computed tomography (CT) and angiography. It is characterized by iatrogenic acute kidney injury within 24-72 hours after intravascular injection of iodinebased radiocontrast agents (1-3). CIN constitutes 10\% of hospital-acquired acute kidney injuries (2). Moreover, it is associated with prolonged hospital stay, increased cardiovascular and renal morbidity, and all-cause mortality $(4,5)$. On the other hand, it is proposed that renal function can be affected following contrast agent administration even if CIN does not occur. For this reason, it is suggested that attention must be paid to renal function in patients not developing CIN, and that renal functions of patients exposed to contrast agents need to be followed up for a long period $(4,6)$.

Biochemical changes in the serum and urine of people exposed to intravenous contrast medium are generally transient. Proteinuria is one of these changes (4). Proteinuria and $\mathrm{pH}$ changes determined using urine dipstick testing within 24 hours after the administration of the contrast agents mostly occur due to the presence of contrast medium in the urine and should be interpreted with caution $(7,8)$. Although there are studies on the potential analytical interference of contrast media in laboratory diagnostics, it is important to emphasize that sample quality and integrity may also be affected by the problems caused by these compounds (9). 
Proteinuria can be detected by semiquantitative (dipstick urinalysis) or quantitative (24-hour urine protein test or albumin/creatinine $[\mathrm{Alb} / \mathrm{Cr}$ ] ratio in a spot urine samples) tests (10). Dipstick urinalysis is a widely used, conventional, quick and easy method. Urine protein concentrations $>10-20 \mathrm{mg} / \mathrm{dL}$ can be detected with this test (11). However, the dipstick test may give false positive and negative results. Alkaline or concentrated urine may result in false positive results, while acidic or diluted urine may result in false negative results (12). In that case, quantitative tests, 24 -hour urine protein test, or $\mathrm{Alb} / \mathrm{Cr}$ ratio in spot urine, should be performed to verify the results. Spot urine test is preferred, as it is more easily performed in comparison to 24-hour urine test (11). While the spot urine albumin/creatinine $(\mathrm{Alb} / \mathrm{Cr}$ ) ratio is a valid substitute for 24 -hour urine albumin excretion rate, it has its limitations (13).

It has been acknowledged that measurement of serum creatinine as an indicator of kidney dysfunction is not an ideal method and even Modification of Diet in Renal Disease (MDRD) and Cockcroft Gault methods have their limitations. Albuminuria, a known marker for the progression of chronic renal disease, is a direct consequence of glomerular damage and increases in glomerular dysfunction (13). It has been previously shown that contrast agent has toxic renal effects such as enzymuria and proteinuria without causing nephropathy $(7,14)$. In this study, we aimed to quantitatively (using the urinary Alb/Cr ratio) demonstrate the potential effect of a contrast agents, without causing significant renal dysfunction, on glomerular albumin loss, in patients who underwent $\mathrm{CT}$ for various reasons.

\section{MATERIAL AND METHOD}

The study was carried out with the permission of Dışkapı Yıldırım Beyazıt Training and Research Hospital Clinical Research Ethics Committee (Date: 27.03.2017, Decision No:36/28). All procedures were carried out in accordance with the ethical rules and the principles of the Declaration of Helsinki. Written informed consents of all patients were obtained before inclusion. This study was designed as a single-center, prospective observational study.

Among the patients hospitalized in our clinic, those aged between 18 and 75 years, who underwent contrastenhanced CT scanning for any reason (staging of acute pancreatitis, malignancy, investigation of the etiology of anemia, etc.) and patients who did not have any complications (CIN, allergy, etc.) after contrast agent were included in the present study. The patients with NYHA (New York Heart Association) class III-IV heart failure and who had renal dysfunction (abnormal serum creatinine levels and a glomerular filtration rate (GFR) $<60 \mathrm{~mL} / \mathrm{min} / 1.72 \mathrm{~m}^{2}$ ) were not included in the present study. Advanced age ( $>75)$, heart failure and known kidney disease are important risk factors for CIN (5). Therefore, at the beginning of the study, these groups of patients with a high probability to develop CIN, were not included in the study. In the follow-up, patients diagnosed with CIN according to the European Society of Urogenital Radiology (ESUR) guideline were excluded from the study. CIN was defined as the $25 \%$ or $0.5 \mathrm{mg} / \mathrm{dl}$ increase in serum creatinine levels from baseline within 2-3 days after contrast agent administration without the presence of any other etiological causes (3).

The same contrast agent (iohexol) in the same amount $(90 \mathrm{~mL})$ was used in all patients. Due to the presence of at least one risk factor for contrast nephropathy (diabetes mellitus, use of angiotensin converting enzyme inhibitors, angiotensin receptor blockers, or diuretics, advanced age) in all patients of our study, $0.9 \%$ sodium chloride was administered to all patients 6-12 hours before the procedure and in a 12-24 hour period after the procedure via intravenous route at a dose of $1 \mathrm{mg} /$ $\mathrm{kg} /$ hour. In addition, 2x1200 mg N-acetylcysteine was given via oral route. The $\mathrm{Alb} / \mathrm{Cr}$ ratio in spot urine was measured both before the CT procedure (pre-procedure time) and at the $6^{\text {th }}, 12^{\text {th }}, 24^{\text {th }}, 48^{\text {th }}$, and $72^{\text {nd }}$ hours after the CT procedure (post-procedure time). Previous studies have shown that, in CIN pathophysiology, renal damage begins earlier ( $<4$ hours), before the disruption in creatinine levels (15). Therefore, in our study, we preferred to take measurements also at the $6^{\text {th }}, 12^{\text {th }}$, and $24^{\text {th }}$ hour time points. We aimed to compare the values within the different periods according to the time of performing CT procedure (between pre-procedure time value and post-prosedure time values). The hypothesis of this study is not to compare repeated measures in multibl. Urine albumin and creatinine concentrations were evaluated immediately in the central laboratory with a Beckman Coulter AU5800 (CA, USA) instrument, using the turbidimetric method for urine albumin and the kinetic Jaffe procedure for urine creatinine. According to KDIGO (Kidney Disease: Improving Global Outcomes) clinical practice guideline urine albumin-to-creatinine ratio was calculated and the results were divided into 2 categories: (1) normoalbuminuria; $\mathrm{Alb} / \mathrm{Cr}$ ratio $<30$ $\mathrm{mg} / \mathrm{g}$ (group-1); (2) microalbuminuria; $\mathrm{Alb} / \mathrm{Cr}$ ratio $\geq 30$ $\mathrm{mg} / \mathrm{g}$ (group-2) (16).

\section{Statistical Analysis}

Data were analyzed using the PASW Statistics for Windows, version 18.0 (SPSS Inc., Chicago, IL, USA). Normality distribution analysis of the data was performed using Kolmogorov-Smirnov and Shapiro-Wilk tests. Normally distributed numerical data were expressed as mean $\pm S D$, non-normally distributed numerical data were expressed as median (interquartile range, IQR 
25-75\%). Wilcoxon Signed Rank Test was used for dependent group pairwise comparison analysis between pre-procedure measurements and post procedure $6^{\text {th }}$, $12^{\text {th }}, 24^{\text {th }}, 48^{\text {th }}$, and $72^{\text {nd }}$ hour measurements. P value $<0.05$ was considered statistically significant.

\section{RESULTS}

The median age of the 103 participants (55.3\% female) included in the present study was 61(47-71) years. General characteristics of the patients are seen in Table 1. Comparison of $\mathrm{Alb} / \mathrm{Cr}$ ratio at each time point with preprocedure values is seen in Table 2 . Alb/Cr ratio at the post-procedure $72^{\text {nd }}$ hour was significantly lower than the pre-procedure value. There was no significant change in GFR values at any time point. The decrease in urea and creatinine values at only post-procedure $48^{\text {th }}$ hour was statistically significant compared to the pre-procedure values.

When the patients were evaluated for the albuminuria level before the procedure, it has been seen that 73 patients (70.9\%) had an $\mathrm{Alb} / \mathrm{Cr}$ ratio of $<30 \mathrm{mg} / \mathrm{g}$ (group-1) and 30 patients (29.1\%) had an $\mathrm{Alb} / \mathrm{Cr}$ ratio of $\geq 30 \mathrm{mg} / \mathrm{g}$ (group-2). In group-1; it has been observed that the $\mathrm{Alb} / \mathrm{Cr}$ ratio at the post-procedure $6^{\text {th }}, 12^{\text {th }}, 24^{\text {th }}$, and $48^{\text {th }}$ hours was significantly higher than the value at pre-procedure time, while the $\mathrm{Alb} / \mathrm{Cr}$ ratio at the post-

\begin{tabular}{|c|c|}
\hline & $\mathrm{n}=103$ \\
\hline \multicolumn{2}{|l|}{ Gender } \\
\hline Female, n (\%) & $57(55.3)$ \\
\hline Male, n (\%) & $46(44.7)$ \\
\hline Age, years & $61(47-71)$ \\
\hline \multicolumn{2}{|l|}{ The reason for having CT scan } \\
\hline Malignancy, n (\%) & $47(45.6)$ \\
\hline Pancreatitis, n (\%) & $27(26.2)$ \\
\hline Anemia, n (\%) & $15(14.6)$ \\
\hline Mass, n (\%) & $4(3.9)$ \\
\hline Other, n (\%) & $10(9.7)$ \\
\hline Diabetes, n (\%) & $42(40.7)$ \\
\hline Hb1Ac, \%, median (IQR 25-75\%) & $7.2(5.9-11.8)$ \\
\hline Hypertension, n (\%) & $37(35.9)$ \\
\hline \multicolumn{2}{|l|}{ Urinary protein dipstick test before the procedure } \\
\hline Negative & $101(98.1)$ \\
\hline $1+$ & $1(1.0)$ \\
\hline $2+$ & $1(1.0)$ \\
\hline \multicolumn{2}{|l|}{ Pre-procedure } \\
\hline Serum urea, mg/dL, median (IQR 25-75\%) & $29(22-39)$ \\
\hline Serum creatinine, mg/dL, median (IQR 25-75\%) & $0.8(0.7-0.9)$ \\
\hline GFR, $\mathrm{mL} / \mathrm{min} / 1.73 \mathrm{~m}^{2}$, median (IQR $25-75 \%$ ) & $84(70.1-102)$ \\
\hline Urine Alb/Cr ratio, mg/g, median (IQR 25-75\%) & $16.5(7.7-38.2)$ \\
\hline
\end{tabular}

procedure $72^{\text {nd }}$ hour was similar to the value at preprocedure time. In group 2, it has been observed that $\mathrm{Alb} / \mathrm{Cr}$ ratio values at all post-procedure time except the $24^{\text {th }}$ hour were statistically significantly lower than the values at the pre-procedure time (Table 3 ).

The percentage of diabetic patients was similar in the groups with and without pre-procedure microalbuminuria $(\mathrm{p}=0.24) ; 27(37.5 \%)$ patients in the group with an $\mathrm{Alb} / \mathrm{Cr}$ ratio $<30$ and $15(50 \%)$ patients in the group with an $\mathrm{Alb} / \mathrm{Cr}$ ratio $\geq 30$ had diabetes.

Table 2. The comparison of changes in the albumin/creatinine ratio between each specific time point of the post-procedure time and the pre-procedure time

\begin{tabular}{|c|c|c|}
\hline Alb/Cr ratio $(n=103)$ & median (IQR 25-75\%) & $\mathbf{p}^{*}$ \\
\hline Pre-procedure & $16.5(7.7-38.2)$ & \multirow{2}{*}{0.37} \\
\hline Post-procedure $6^{\text {th }}$ hour & $15.7(8-38.2)$ & \\
\hline Pre-procedure & $16.5(7.7-38.2)$ & \multirow{2}{*}{0.15} \\
\hline Post-procedure $12^{\text {th }}$ hour & $15.6(9.3-28.8)$ & \\
\hline Pre-procedure & $16.5(7.7-38.2)$ & \multirow{2}{*}{0.79} \\
\hline Post-procedure $24^{\text {th }}$ hour & $15.9(9-33)$ & \\
\hline Pre-procedure & $16.5(7.7-38.2)$ & \multirow{2}{*}{0.23} \\
\hline Post-procedure $48^{\text {th }}$ hour & $16.5(9.1-33.3)$ & \\
\hline Pre-procedure & $16.5(7.7-38.2)$ & \multirow{2}{*}{0.046} \\
\hline Post-procedure $72^{\text {nd }}$ hour & $15.1(8-27.2)$ & \\
\hline
\end{tabular}

Table 3. The comparison of changes in the albumin/creatinine ratio of patients without microalbuminuria (group-1) and with microalbuminuria (group-2) at the specific time point of the postprocedure time and the pre-procedure time.

\begin{tabular}{|c|c|c|c|}
\hline & & $\begin{array}{c}\text { median (IQR } \\
25-75 \%)\end{array}$ & $\mathbf{p}^{*}$ \\
\hline Group-1 (Alb/Cr ratio <30) & $n=73$ & & \\
\hline Pre-procedure & & $9.8(6.9-18)$ & \multirow{2}{*}{0.046} \\
\hline Post-procedure $6^{\text {th }}$ hour & & $13(6.9-21.7)$ & \\
\hline Pre-procedure & & $9.8(6.9-18)$ & \multirow{2}{*}{0.021} \\
\hline Post-procedure $12^{\text {th }}$ hour & & $12.8(8.6-20.9)$ & \\
\hline Pre-procedure & & $9.8(6.9-18)$ & \multirow{2}{*}{0.020} \\
\hline Post-procedure $24^{\text {th }}$ hour & & $12.6(7.4-24.9)$ & \\
\hline Pre-procedure & & $9.8(6.9-18)$ & \multirow{2}{*}{0.040} \\
\hline Post-procedure $48^{\text {th }}$ hour & & $12.7(6.8-21.7)$ & \\
\hline Pre-procedure & & $9.8(6.9-18)$ & \multirow{2}{*}{0.79} \\
\hline Post-procedure $72^{\text {nd }}$ hour & & $10.7(7.3-18)$ & \\
\hline Group-2 (Alb/Cr ratio $\geq 30$ ) & $\mathrm{n}=\mathbf{3 0}$ & & \\
\hline Pre-procedure & & $58(41.7-166.1)$ & \multirow{2}{*}{0.002} \\
\hline Post-procedure $6^{\text {th }}$ hour & & $41.4(23.1-108.6)$ & \\
\hline Pre-procedure & & $58(41.7-166.1)$ & \multirow{2}{*}{$<0.001$} \\
\hline Post-procedure $12^{\text {th }}$ hour & & $30.2(19.6-99.8)$ & \\
\hline Pre-procedure & & $58(41.7-166.1)$ & \multirow{2}{*}{0.12} \\
\hline Post-procedure $24^{\text {th }}$ hour & & $42.9(22.7-112.2)$ & \\
\hline Pre-procedure & & $58(41.7-166.1)$ & \multirow{2}{*}{0.002} \\
\hline Post-procedure $48^{\text {th }}$ hour & & $39.9(29.8-108.1)$ & \\
\hline Pre-procedure & & $58(41.7-166.1)$ & \multirow{2}{*}{0.009} \\
\hline Post-procedure $72^{\text {nd }}$ hour & & $43(19-115)$ & \\
\hline
\end{tabular}




\section{DISCUSSION}

The main finding of our study is that it has been seen that there is a significant increase in the urinary $\mathrm{Alb} /$ $\mathrm{Cr}$ ratio in patients with $\mathrm{Alb} / \mathrm{Cr}$ ratio $<30 \mathrm{mg} / \mathrm{g}$ after the use of nonionic low osmolar contrast material (Iohexol) used for CT, but this increase does not reach the level of microalbuminuria. It should be kept in mind that there may be changes in Alb/Cr ratio values in patients receiving contrast agent for $\mathrm{CT}$ procedures, even if complications such as CIN have not developed in these patients.

Besides the benefits of contrast agents commonly used in daily radiology practice, they also have undesirable effects. Intravenous contrast media use is sometimes necessary for diagnosis but mostly associated with transient biochemical irregularities. In a study by Okoye et al. (7), a significant decrease in serum sodium and potassium levels, a significant increase in serum urea, $\mathrm{Cr}$, and urine $\mathrm{pH}$ were detected after exposure to the contrast agent, and these changes were reported to return to normal 72 hours after contrast agent exposure. In our study, it has been seen that the decrease in urea and creatinine values at only post-procedure 48th hour was statistically significant compared to the values at pre-procedure time. This was attributed to the hydration of all patients starting from before the procedure and lasting until the 48th hour after the procedure.

In addition, even without causing renal dysfunction, the contrast agent has many renal impacts such as proteinuria, enzymuria, and increased urinary $\mathrm{pH}$ $(14,17)$. In their study investigating the effect of exposure to nonionic radiocontrast agents on microalbuminuria in patients undergoing angiography, Chu et al. (18) reported that ordinary dose low osmolar contrast agents had no significant effect on the presence and level of microalbuminuria. Again, as a result of a series of studies conducted by Holtas et al. $(19,20)$, it was suggested that the actual cause of proteinuria observed after renal angiography may be due to the chemical structure of the contrast agent used and individual factors. The same researchers proposed that osmolality is not a dominant factor; and that proteinuria may occur due to the destruction in the kidney and vascular endothelium caused by the contrast media $(19,20)$.

Similar to the study of Chu et al. (18), we found that there was no significant increase in $\mathrm{Alb} / \mathrm{Cr}$ ratio after the procedure. The reason for these findings may be the use of low-dose contrast media in all patients and ensuring adequate hydration starting from before the procedure. However, when we divided the patients into groups according to the presence of microalbuminuria, we obtained different results. In the 73 patients constituting group-1; it has been observed that there were significant increases in $\mathrm{Alb} / \mathrm{Cr}$ ratio in all of the repeated measurements up to the post-procedure 48th hour compared to the values at the pre-procedure time, but these increases did not reach the microalbuminuria level. Since we preferred to use the $\mathrm{Alb} / \mathrm{Cr}$ ratio, which gives quantitative results, rather than the dipstick test, which is known to give false positive results in post-procedure albuminuria evaluations; we think that the contrast agent disrupts the permeability by causing temporary damage to the glomerular endothelium and causes a slight, transient increase in albuminuria.

In the 30 patients constituting group-2, it has been observed that $\mathrm{Alb} / \mathrm{Cr}$ ratio at pre-procedure time was significantly lower than all post-procedure time measurements except the $24^{\text {th }}$ hour measurement. This may be attributed to the fact that patients with microalbuminuria are not able to reflect the transiently increasing albuminuria due to the impaired glomerular membrane structure. Microalbuminuria occurs as a result of impaired glomerular permselectivity to plasma proteins, and whether there is a decrease in glomerular filtration rate, the presence of long-term microalbuminuria in the patient is defined as chronic kidney disease according to the guidelines prepared by the National Kidney Foundation - Kidney Disease Outcomes Quality Initiative (NKF-KDOQI) (21). As in patients with microalbuminuria, the elimination time of the contrast agent is prolonged in individuals with impaired renal function (22). We believe that the osmotic diuretic effect will be more pronounced in these patients, and the $\mathrm{Alb} / \mathrm{Cr}$ ratio may appear low. In addition, it should not be overlooked that compared to the normoalbuminuric group, our group was not large enough to evaluate the effect of contrast agents in microalbuminuric patients.

Our study has some limitations. Firstly, low-dose contrast agent was used in all patients. Secondly, we did not measure other specific urinary proteins (e.g.; transferrin or $\beta 2$-microglobulin) or tubular enzymes. Finally, since we excluded patients with high risk for developing CIN, the results of our study are not valid for these patients.

\section{CONCLUSION}

In the present study, it has been shown that contrast material exposure during CT procedures may have negligible toxic effects on glomerular albumin loss and the changes in $\mathrm{Alb} / \mathrm{Cr}$ ratio are transient. The results of the present study support our hypothesis that not only tubular damage but also glomerular damage may occur due to the use of contrast agents. Our study should be considered as a pilot study and further studies are needed to reveal the clinical significance of the transient increase in $\mathrm{Alb} / \mathrm{Cr}$ ratio due to the contrast agent. 


\section{ETHICAL DECLARATIONS}

Ethics Committee Approval: The study was carried out with the permission of Dişkapı Yıldırım Beyazıt Training and Research Hospital Clinical Research Ethics Committee (Date: 27.03.2017, Decision No: 36/28).

Informed Consent: All patients signed the free and informed consent form.

Referee Evaluation Process: Externally peer-reviewed.

Conflict of Interest Statement: The authors have no conflicts of interest to declare.

Financial Disclosure: The authors declared that this study has received no financial support.

Author Contributions: All of the authors declare that they have all participated in the design, execution, and analysis of the paper, and that they have approved the final version.

\section{REFERENCES}

1. Andreucci M, Faga T, Pisani A, Sabbatini M, Russo D, Michael A. Prevention of contrast-induced nephropathy through a knowledge of its pathogenesis and risk factors. ScientificWorldJournal 2014; 2014: 823169 .

2. Deek H, Newton P, Sheerin N, Noureddine S, Davidson PM. Contrast media induced nephropathy: a literature review of the available evidence and recommendations for practice. Aus Crit Care 2014; 27: 166-71.

3. Stacul F, van der Molen AJ, Reimer P, et al. Contrast induced nephropathy: updated ESUR Contrast Media Safety Committee guidelines. Eur Radiol 2011; 21: 2527-41.

4. Katsiki N, Athyros VG, Karagiannis A, Mikhailidis DP. ContrastInduced Nephropathy: An "All or None" Phenomenon? Angiology 2015; 66: 508-13.

5. Karadeniz M, Kandemir H, Sarak T, Alp Ç. Akut koroner sendromda perkütan koroner girişim yapılan hastalarda kontrast nefropati prevalansı. J Health Sci Med 2018; 1: 34-9.

6. Ronco F, Tarantini G, McCullough PA. Contrast induced acute kidney injury in interventional cardiology: an update and key guidance for clinicians. Rev Cardiovasc Med 2020; 2: 9-23.

7. Okoye O, Ikubor J, Okosun RE. Biochemical changes associated with intravenous use of contrast media. Tropical J Nephrol 2015; 10: 73-8.

8. Seeliger E, Persson PB. Kidney damage by iodinated contrast media. Acta Physiol (Oxf.) 2019; 227: e13259.

9. Lippi G, Daves M, Mattiuzzi C. Interference of medical contrast media on laboratory testing. Biochemia Medica 2014; 24: 80-8.

10. Keane WF, Eknoyan G. Proteinuria, albuminuria, risk, assessment, detection, elimination (PARADE): a position paper of the National Kidney Foundation. Am J Kidney Dis 1999; 33: 1004-10.

11. Thorp ML. Diabetic nephropathy: common questions. Am Fam Physician 2005; 72: 96-9.

12. Simerville JA, Maxted WC, Pahira JJ. Urinalysis: a comprehensive review. Am Fam Physician 2005; 71: 1153-62.

13.Levin A, Pate GE, Shalansky S, et al. N-acetylcysteine reduces urinary albumin excretion following contrast administration: evidence of biological effect. Nephrol Dial Transplant 2007; 22: 2520-4.

14. Nicot GS, Merle LJ, Charmes JP, et al. Transient glomerular proteinuria, enzymuria, and nephrotoxic reaction induced by radiocontrast media. JAMA 1984; 252: 2432-4.
15. Tumlin JA, Wang A, Murray PT, Mathur VS. Fenoldopam mesylate blocks reductions in renal plasma flow after radiocontrast dye infusion: a pilot trial in the prevention of contrast nephropathy. Am Heart J 2002; 143: 894-903.

16. Inker LA, Astor BC, Fox CH, et al. KDOQI US commentary on the 2012 KDIGO clinical practice guideline for the evaluation and management of CKD. Am J Kidney Dis 2014; 63: 713-35.

17. Bachorzewska-Gajewska H, Malyszko J, Sitniewska E, Malyszko JS, Dobrzycki S. Neutrophil gelatinase-associated lipocalin (NGAL) correlations with cystatin C, serum creatinine and eGFR in patients with normal serum creatinine undergoing coronary angiography. Nephrol Dial Transplant 2007; 22: 295-6.

18. Chu CS, Cheng KH, Lee KT, et al. Negligible effect of non-ionic radiocontrast medium exposure on microalbuminuria in patients undergoing coronary angiography. Int J Cardiol 2009; 132: 150-1.

19. Holtås S, Billström A, Tejler L. Proteinuria following nephroangiography. IX. Chemical and morphological analysis in dogs. Acta Radiol Diagn 1981; 22: 427-33.

20.Holtås S, Golman K, Törnquist C. Proteinuria following nephroangiography. VIII. Comparison between diatrizoate and iohexol in the rat. Acta Radiol Suppl 1980; 362: 53-5.

21.Hogg RJ, Furth S, Lemley KV, et al. National Kidney Foundation's Kidney Disease Outcomes Quality Initiative clinical practice guidelines for chronic kidney disease in children and adolescents: evaluation, classification, and stratification. Pediatrics 2003; 111: 1416-21

22. Thomsen HS, Morcos SK. Radiographic contrast media. BJU Int 2000; 86: 1-10. 